\title{
Letter to the EFSUMB Newsletter Editor Adrian Săftoiu
}

In the first issue from 2013 of Ultraschall in der Medizin, I read an interesting paper (EFSUMB Executive Bureau. Ultraschall in Med 2013; 34: 92), regarding a new classification of the ultrasound method.

The latest developments of this now "old technique", with more than 50 years of clinical use, made possible two things: firstly, development of very sophisticated new ultrasound machines (with a lot of technological capabilities such as Contrast Enhanced Ultrasonography, Elastography, Fusion capabilities and others), and on the other hand, small, compact, portable ultrasound systems (very recently with wireless connection of the probe). Of course, the results of ultrasound examinations

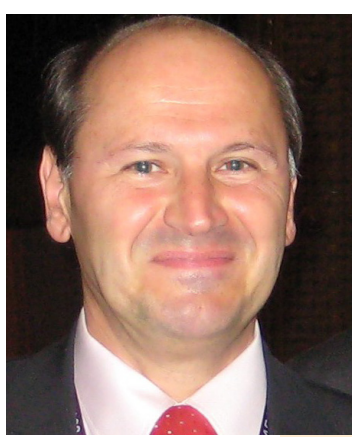

Prof loan Sporea

performed with these two types of ultrasound machines cannot be similar. 
The initiative of EFSUMB's Executive Bureau to split the evaluation with ultrasound systems into three categories (Echoscopy; Point of care ultrasound; Echography or ultrasonography) seems to be a good idea. From the start, it defines the purpose of the examination and also, the level of performance. Echoscopy means answering to a punctual question such as: does the patient have or not ascites; is it or not an obstructive jaundice; does the patient have an aortic aneurysm etc. Point of care ultrasound is a clinical ultrasound evaluation, starting from data obtained from the patient and using the result of ultrasound examination to make decisions regarding diagnosis and treatment. Finally, ultrasonography means a comprehensive and full examination (such as abdominal ultrasonography) with a final report to be delivered, including stored images of the examined organs.

I have only one comment regarding "point of care ultrasound" (C Moore, J Copel. NEJM 2011; 364: 749-57). As I mentioned in a previous paper (I Sporea. Med Ultrason 2012; 14: 3-4), we can discuss which term to use: "point of care ultrasound" or "clinical ultrasound". Because my specialty is a clinical one, and respecting the opinion of a good friend of many of us (I am speaking of Prof. Dr. Lucas Greiner, the initiator of clinical ultrasound), I think that "point of care ultrasound" is in reality a clinical ultrasound. In such situations, the patient is evaluated from a clinical point of view and ultrasound exam is a part of this examination. The aim of the ultrasound exam is to answer to some clinical questions and finally to make decisions regarding diagnosis and treatment. The argument against the term of "clinical ultrasound" can be that Echoscopy is also a clinical ultrasonography, but with a limited area of evaluation.

Finally, I want to express my support for this new, practical classification of the ultrasound evaluation. Knowing what type of examination is performed, we know what we can expect from it, depending on the ultrasound machine used and how trained is the person performing it.

\section{Prof Ioan Sporea}

Department of Gastroenterology and Hepatology

University of Medicine and Pharmacy

“Victor Babeș” Timișoara Romania 